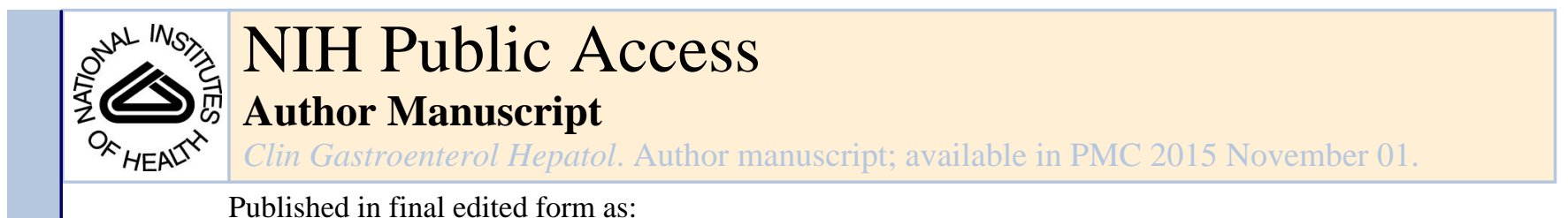

Published in final edited form as:

Clin Gastroenterol Hepatol. 2014 November ; 12(11): 1840-1847.e1. doi:10.1016/j.cgh.2014.04.034.

\title{
Durability and Predictors of Successful Radiofrequency Ablation for Barrett's Esophagus
}

\author{
Sarina Pasricha ${ }^{1}$, William J. Bulsiewicz ${ }^{1}$, Kelly E. Hathorn ${ }^{1}$, Srinadh Komanduri ${ }^{2}$, V. Raman \\ Muthusamy $^{3}$, Richard I. Rothstein ${ }^{4}$, Herbert C. Wolfsen ${ }^{5}$, Charles J. Lightdale ${ }^{6}$, Bergein F. \\ Overholt $^{7}$, Daniel S. Camara ${ }^{8}$, Evan S. Dellon ${ }^{1}$, William D. Lyday ${ }^{9}$, Atilla Ertan ${ }^{10}$, Gary W. \\ Chmielewski $^{11}$, and Nicholas J. Shaheen ${ }^{1}$ \\ ${ }^{1}$ University of North Carolina at Chapel Hill, Center for Esophageal Diseases and Swallowing, \\ Division of Gastroenterology and Hepatology, Chapel Hill, NC \\ ${ }^{2}$ Northwestern University School of Medicine, Chicago, IL \\ ${ }^{3}$ David Geffen School of Medicine at UCLA, Los Angeles, CA \\ ${ }^{4}$ Dartmouth University School of Medicine, Hanover, $\mathrm{NH}$ \\ ${ }^{5}$ Mayo Clinic Florida, Jacksonville, FL \\ ${ }^{6}$ Columbia University School of Medicine, New York, NY \\ ${ }^{7}$ Gastrointestinal Associates, Knoxville, TN
}

\begin{abstract}
(C) 2014 The American Gastroenterological Association. Published by Elsevier Inc. All rights reserved.
Corresponding Author: Nicholas J. Shaheen, MD, MPH Professor of Medicine and Epidemiology Director, Center for Esophageal Diseases and Swallowing University of North Carolina School of Medicine CB\#7080 Chapel Hill, NC 27599-7080 Phone: (919) 966-2513 Fax: (919) 843-2508 .

Publisher's Disclaimer: This is a PDF file of an unedited manuscript that has been accepted for publication. As a service to our customers we are providing this early version of the manuscript. The manuscript will undergo copyediting, typesetting, and review of the resulting proof before it is published in its final citable form. Please note that during the production process errors may be discovered which could affect the content, and all legal disclaimers that apply to the journal pertain.
\end{abstract}

The abstract of this work was previously presented in the Presidential Plenary of the AGA on May 18, 2013, in Orlando, FL. It has not been presented or published elsewhere.

Author Contributions: Sarina Pasricha: study concept and design, acquisition of data, analysis and interpretation of data, drafting of the manuscript, critical revision of the manuscript for important intellectual content, statistical analysis; William J. Bulsiewicz: study concept and design, acquisition of data, analysis and interpretation of data, drafting of the manuscript, critical revision of the manuscript for important intellectual content, statistical analysis; Kelly E. Hathorn: study concept and design, analysis and interpretation of data, drafting of the manuscript, critical revision of the manuscript for important intellectual content; Srinadh Komanduri: study concept, acquisition of data, interpretation of data, critical revision of the manuscript for important intellectual content; Raman V. Muthusamy: study concept, acquisition of data, interpretation of data, critical revision of the manuscript for important intellectual content; Richard I. Rothsteinः study concept, acquisition of data, interpretation of data, critical revision of the manuscript for important intellectual content; Herbert C. Wolfsen" study concept, acquisition of data, interpretation of data, critical revision of the manuscript for important intellectual content; Charles J. Lightdale: study concept, acquisition of data, interpretation of data, critical revision of the manuscript for important intellectual content; Bergein F. Overholt ${ }^{\star}$ study concept, acquisition of data, interpretation of data, critical revision of the manuscript for important intellectual content; Daniel S. Camara: study concept, acquisition of data, interpretation of data, critical revision of the manuscript for important intellectual content; Evan S. Dellonः study concept, acquisition of data, interpretation of data, critical revision of the manuscript for important intellectual content; William D. Lyday: study concept, acquisition of data, interpretation of data, critical revision of the manuscript for important intellectual content; Atilla Ertan: study concept, acquisition of data, interpretation of data, critical revision of the manuscript for important intellectual content; Gary W. Chmielewski: study concept, acquisition of data, interpretation of data, critical revision of the manuscript for important intellectual content; Nicholas J. Shaheen: study concept and design, analysis and interpretation of data, critical revision of the manuscript for important intellectual content, study supervision.

The other authors have no conflicts to declare. 
${ }^{8}$ Digestive Health Physicians, Cheektowaga, NY

${ }^{9}$ Atlanta Gastroenterology Associates, Atlanta GA

10University of Texas School of Medicine, Houston, TX

${ }^{11}$ Rush Medical College, Chicago, IL

\section{Abstract}

Background \& Aims-Following radiofrequency ablation (RFA), patients may experience recurrence of Barrett's esophagus (BE) after complete eradication of intestinal metaplasia (CEIM). Rates and predictors of recurrence after successful eradication are poorly described.

Methods-We used the U.S. RFA Registry, a nationwide registry of BE patients receiving RFA, to determine rates and factors that predicted recurrence of IM. We assessed recurrence by KaplanMeier analysis for the overall cohort and by worst pretreatment histology. Characteristics associated with recurrence were included in a logistic regression model to identify independent predictors.

Results-Among 5521 patients, 3728 had biopsies $\geq 12$ months after initiation of RFA. Of these, 3169 (85\%) achieved CEIM, and 1634 (30\%) met inclusion criteria. Average follow-up was 2.4 years after CEIM. IM recurred in 334 (20\%), and was non-dysplastic or indefinite for dysplasia in $86 \%$ (287/334); the average length of recurrent BE was $0.6 \mathrm{~cm}$. In Kaplan-Meier analysis, more advanced pretreatment histology was associated with an increased yearly recurrence rate. Compared to patients without recurrence, patients with recurrence were more likely, based on bivariate analysis, to be older, have longer BE segments, be non-Caucasian, have dysplastic BE before treatment, and require more treatment sessions. In multivariate analysis, likelihood for recurrence was associated with increasing age and $\mathrm{BE}$ length, and non-Caucasian race.

Conclusion-BE recurred in $20 \%$ of patients followed for an average of 2.4 years after CEIM. Most recurrences were short segments and were non-dysplastic or indefinite for dysplasia. Older age, non-Caucasian race, and increasing length of BE length were all risk factors. These risk factors should be considered when planning post-RFA surveillance intervals.

\section{Keywords}

esophageal cancer; risk factors; adenocarcinoma; EAC

Barrett's esophagus (BE) affects 1-2\% of the general population and is associated with an increased risk of esophageal adenocarcinoma (EAC), a cancer with increasing incidence over the last four decades. ${ }^{1-5} \mathrm{BE}$ is defined as an endoscopically visible metaplastic change of the esophagus in which the squamous epithelium is replaced by columnar mucosa exhibiting goblet cells, also termed specialized intestinal metaplasia (IM). Endoscopic ablative therapy is a treatment modality aimed at eradicating dysplasia and intestinal metaplasia to prevent the development of EAC. ${ }^{6}$

Radiofrequency ablation (RFA) has been shown to be safe and effective in treating BE, with substantial rates of complete eradication of dysplasia (CED) and intestinal metaplasia (CEIM) and decreased rates of progression to EAC in the setting of dysplasia. ${ }^{7-9}$ However, 
the long-term durability of the neosquamous epithelium remains poorly characterized. The few studies reporting durability of CED and CEIM are limited by small sample sizes, and most have short duration of follow-up. Also, these studies have unclear generalizability, as they were conducted at tertiary care centers. ${ }^{10-15}$

The aim of this study was to investigate the rate of recurrence of intestinal metaplasia after successful CEIM in a nationwide, multicenter registry of patients treated with RFA. We also sought to define risk factors for recurrence of $\mathrm{BE}$ after successful ablation.

\section{Methods}

\section{U.S. RFA Patient Registry}

The U.S. RFA Patient Registry is a multi-center collaboration reporting processes and outcomes of care for patients treated with RFA for BE at 148 institutions in the U.S. (113 community-based, 35 academic-affiliated). The registry was developed as a research tool to monitor clinical outcomes after RFA using the HALO Ablation Systems (Covidien, GI Solutions, Sunnyvale, CA), and is funded by Covidien. The registry does not mandate protocols for care, but provides a suggested protocol for treatment and follow-up of patients with BE. All physicians participating $(n=320)$ either used Western institutional review board (IRB) approval, or obtained IRB approval through their respective institutions.

\section{Registry Patient Eligibility}

Patients were enrolled from July, 2007 to July, 2011. Patients were eligible for inclusion if: (1) they had endoscopic evidence of columnar metaplasia in the tubular esophagus with accompanying biopsies demonstrating intestinal metaplasia, and (2) received RFA for BE. Subjects were classified using standardized histological grading, including non-dysplastic $\mathrm{BE}$ (NDBE), indefinite for dysplasia (IND), low-grade dysplasia (LGD), high-grade dysplasia (HGD), or carcinoma, subclassified as intramucosal carcinoma (IMC), and invasive esophageal adenocarcinoma (EAC). ${ }^{16,17}$ Patients who had received one or more RFA treatments prior to enrollment had collection of retrospective data, with subsequent prospective collection for ensuing visits. Patients who had not yet undergone treatment were prospectively enrolled in the study.

\section{Data Collection and Record Retention}

Information collected in the registry includes demographic data, histology prior to treatment, endoscopic findings, number of treatment sessions, ablation outcomes, and complications. All data were recorded on standardized case report forms. Data were collated into a central electronic database, with real-time monitoring for logic checks and consistency. Data were analyzed by investigators in the clinical epidemiology program at UNC (T32 DK07634), who had complete access to the data.

\section{Treatment Protocol}

Data from previous clinical trials were given as a guideline for treatment and follow-up protocol. However, because this is a registry study, physicians could deviate from the protocols, depending on specific patient requirements and physician preferences. The 
suggested treatment protocol provided to physician investigators has been previously described. ${ }^{18}$

At enrollment, each patient was interviewed and a Baseline Encounter Form, which collected medical history information and demographics, was completed. The standardized protocol included medical therapy with twice-daily PPIs to minimize any baseline inflammatory changes of the esophageal mucosa and decrease acid reflux prior to and throughout RFA treatment, unless the patient had a documented history of antireflux surgery.

At the initial visit, patients were treated with one of two ablation devices: the HALO 360 circumferential ablation system or the $\mathrm{HALO}^{90}$ focal ablation system. The decision as to initial treatment modality was based on the burden of disease (Barrett's segments of $>3 \mathrm{~cm}$ are generally best treated with the circumferential catheter), as well as operator preference. Recommended treatment protocols were based on previously published data. ${ }^{9}$

\section{Follow-Up Protocol}

Recommendations for the first follow-up visit, which was 2-3 months after treatment initiation, included additional circumferential or focal RFA treatment for any visible residual $\mathrm{BE}$, depending on the extent of the disease. If no visible $\mathrm{BE}$ was observed, four-quadrant biopsies every $\mathrm{cm}$ were recommended throughout the length of the pre-treatment $\mathrm{BE}$. If these biopsies were clear of $\mathrm{BE}$ on pathologic review, the patients entered the surveillance phase. Initial surveillance was recommended at 3 months for HGD or 6 months for NDBE, IND, or LGD. If follow-up biopsies revealed IM or dysplasia, recurrent treatment with RFA was recommended.

Adverse events were reported using standardized forms and terminology. Each site also complied with reporting guidelines for their institution regarding reporting adverse events to their IRB and FDA under the MDR reporting regulation in 21 C.F.R. Part 803.

\section{Outcomes and Statistical Analysis}

For this analysis, our cohort was restricted to subjects who achieved CEIM and had at least one post-CEIM biopsy session. CEIM was defined as an esophageal biopsy session in which no biopsy demonstrated intestinal metaplasia, occurring at least 12 months after initial treatment. Recurrence was defined as any biopsy from the esophagus demonstrating intestinal metaplasia during surveillance. The primary outcome was recurrence of IM. Additionally, to assess differences in outcome using a more stringent definition of CEIM, we also calculated recurrence of IM in patients who had 2 consecutive biopsy sessions with CEIM.

Statistical analysis was performed using Stata software (version 12.0; StataCorp LP, College Station, TX). For descriptive statistics, mean and standard deviations were reported for continuous variables, and percentages were reported for categorical variables. Outcomes are described for all patients, as well as stratified by pre-ablation histology. We used log-rank test to evaluate for differences by worst pre-treatment histology, and compared rates of recurrence and time to recurrence by pre-treatment histology using Pearson's chi-square test 
and one-way analysis of variance, respectively. We performed Kaplan-Meier analysis to determine rates of recurrence of IM. Patients with and without recurrence were compared using parametric statistics for demographic data, pre-treatment EGD findings (length of BE, presence of dysplasia, history of fundoplication) and number of treatments necessary to achieve CEIM. Characteristics associated with recurrence on bivariate analysis $(\mathrm{p}<0.20)$ were included in a logistic regression model to identify independent predictors of recurrence. The model was reduced using the likelihood ratio test.

\section{Results}

A total of 5,521 patients with BE were enrolled in the U.S. RFA Patient Registry and were treated with RFA. Among these patients, 3,728 had biopsies obtained $\geq 12$ months following initiation of RFA therapy. Of these, 3,169 (85\%) achieved CEIM on biopsies obtained $\geq 12$ months after initial treatment, and $559(15 \%)$ had such biopsies taken but did not achieve CEIM. Of the 3,169 who achieved CEIM and had biopsies taken $\geq 12$ months after initial treatment, 1,634 (52\%) had at least 1 additional biopsy session after the biopsy session demonstrating CEIM (Figure 1), and could be analyzed for BE recurrence. Baseline characteristics of the 1,634 patients who met inclusion criteria for analysis are reported in Table 1 . The mean age was 61.7 years, $74 \%$ were male, and $93 \%$ were Caucasian. Among the 1,634 patients included, 66\% $(1,079)$ were enrolled prospectively and $34 \%(555)$ were enrolled with some retrospective data. Pre-treatment histology included: $668(41 \%)$ NDBE, 114 (7\%) IND, 323 (20\%) LGD, 416 (25\%) HGD, 113 carcinomas (92 (6\%) IMC; 21 (1\%) EAC). The mean pre-treatment BE length was $4.0 \mathrm{~cm}$. On average, patients were observed for $2.4 \pm 1.3$ years (3983 total years) after CEIM with $4.3 \pm 1.8$ biopsy sessions performed after CEIM was achieved (7066 total).

Among the 1,634 patients in our study population, recurrence of IM occurred in 334 (20\%). An additional 28 subjects (2\%) had empiric retreatment with RFA for suspected recurrence based on endoscopic appearance, without histological confirmation. Mean length of BE at recurrence was $0.6 \mathrm{~cm}$. When we applied the alternative, more stringent definition of CEIM ( 2 consecutive biopsy sessions without intestinal metaplasia) the rate of recurrence of IM did not markedly change (supplemental table 1). With this more stringent definition, recurrence was seen in $16 \%$ of patients (97/604), with a mean follow-up of $1.8 \pm 1.0$ years (1077 total years; supplemental figures 1 and 2).

In patients undergoing endoscopic surveillance following CEIM, an analysis of durability was performed using Kaplan-Meier estimation (Figure 2a). Subjects with baseline dysplasia demonstrated increased recurrence rates compared to those without dysplasia $(\mathrm{p}<0.001)$ (Figure 2b).

To assess the relationship of pre-treatment histology to the risk of recurrence, recurrence rates were stratified by pre-treatment histology (table 2). More advanced pre-treatment histology was associated with increased yearly recurrence rates (7\% NDBE; 11\% LGD; $10 \%$ HGD; $12 \%$ IMC; $19 \%$ EAC; $p=0.0003$ ), although the rates of recurrence among LGD, HGD and IMC were similar. We also assessed the relationship between pre-treatment histology and histology at the time of BE recurrence (table 3). Overall, histology at 
recurrence was non-dysplastic or IND in 287/334 (86\%). Of patients with recurrence, $94 \%$ (314/334) showed histology identical to or less severe than their baseline histology. In total, $6 \%(20 / 334)$ of patients with recurrence (1.2\% of the overall study population) had histologic disease progression (i.e., histologic grade of recurrence worse than pre-treatment grade). No patient with baseline NDBE or IND progressed to carcinoma. Four patients with LGD, IND or NDBE before treatment progressed to HGD. Two patients with LGD and six patients with HGD before treatment progressed to carcinoma, with all of these being IMC. The annual rate of IM recurrence was $9 \%$ among all 1634 patients included in the analysis, regardless of baseline histology.

Compared to patients without recurrence, patients with recurrence were more likely in bivariate analysis to: 1 ) be older (63.3 vs. $61.3 \mathrm{yrs}, \mathrm{p}=0.003) ; 2$ ) have longer BE (4.8 vs. 3.8 $\mathrm{cm}, \mathrm{p}<0.0001) ; 3$ ) be of non-Caucasian race (93\% vs. $96 \%$, p 0.03); 4) have dysplastic BE (IND or worse) prior to treatment ( $64 \%$ vs. $58 \%, \mathrm{p}=0.03$ ); and, 5) have more total RFA treatment sessions ( 3.1 vs. $2.8, \mathrm{p}=0.004)$. In multivariate analysis, non-Caucasian race (OR $2.00,95 \%$ CI $1.20-3.34)$, length of BE (1.10 per $\mathrm{cm}, 1.06-1.15)$, and age (1.02 per year, 1.01 - 1.03) were independent predictors of IM recurrence (Table 4). Likelihood for recurrence was not influenced by sex, pre-treatment dysplasia, treatment with EMR, or treatment at an academic versus community-based practice in multivariable analysis. Additionally, although total number of treatment sessions was associated with increased risk of recurrence in bivariate analysis, number of RFA sessions was not an independent predictor of IM recurrence in multivariable analysis.

\section{Discussion}

In the largest reported cohort of patients treated with RFA for BE, we investigated the rate of IM recurrence, and predictors for recurrence of IM. Of the 1,634 subjects included in our analysis, 1,300 (80\%) maintained CEIM and $334(20 \%)$ had recurrence of IM. Histology at time of recurrence was NDBE or IND in the vast majority (86\%). However, $6 \%(20 / 334)$ had histological disease progression at time of recurrence. The total length of $\mathrm{BE}$ at the time of recurrence (mean of $0.6 \mathrm{~cm}$ ) was markedly less than baseline (mean of $4.0 \mathrm{~cm}$ ). More advanced pre- treatment histology was associated with an increased yearly recurrence rate. Although the Kaplan-Meier analysis and the bivariate analysis suggested that patients with dysplastic BE prior to treatment were more likely to have recurrence, after controlling for multiple variables, including $B E$ length, this association was insignificant in the multivariate model. The likelihood of recurrence after successful treatment with RFA was increased in those who are older, not Caucasian, and had longer BE segments. These results support the need for long-term surveillance endoscopy even in patients successfully treated for BE with RFA.

Multiple studies have reported the durability of RFA, with differing results. In a recent systematic review and meta-analysis, Orman et al. found that these studies reported an IM recurrence rate of approximately $13 \%$. In this analysis, higher-quality studies had lower recurrence rates $(11 \%)$ than lower-quality studies $(17 \%) .{ }^{19}$ Recent prospective cohorts demonstrate the range of outcomes reported. For example, Phoa and colleagues reported excellent results, with $90 \%$ of 54 patients with neoplastic BE treated with RFA still clear of

Clin Gastroenterol Hepatol. Author manuscript; available in PMC 2015 November 01. 
IM at 5 years. ${ }^{20}$ Conversely, patients in the UK National Halo RFA Registry experienced relatively high recurrence rates, with $38 \%$ of the 335 patients with neoplastic BE demonstrating recurrence of $\mathrm{BE}$ at 12 months. ${ }^{21}$ Our research differs from these prior studies by including patients with a range of pre-treatment histology, as well as patients from both academic and community centers, making our results more generalizable. Additionally, our cohort is the largest to report outcomes associated with radiofrequency ablation.

The cause for the considerable heterogeneity in the reported durability of ablation is unclear. Some of the variability may be due to diverse patient populations, different follow-up time periods, and variability in treatment and surveillance protocols. While the results reported in this registry suggest a higher rate of recurrence than some of the studies noted above, this may in part be a reflection of the non-standardized nature of the treatment protocols, or the lack of inclusion/exclusion criteria used in clinical trials to select optimal patient populations. Additionally, since biopsy protocols after CEIM could not be mandated, some of these "recurrences" may represent incomplete initial treatment. A lack of consensus definition of CEIM may also contribute to the discrepancies in durability outcomes seen in the RFA studies. There is variability in defining CEIM using one negative biopsy session compared to two negative biopsy sessions. In order to understand the impact of alternative definitions of CEIM, we analyzed our cohort using both candidate definitions. Interestingly, we found little difference in recurrence rates based on definition of CEIM (20\% using one negative biopsy session compared to $16 \%$ using two negative biopsy sessions). The most preferable definition for CEIM remains unclear.

Although several studies have reported the durability of RFA, there are very limited data on the predictors of recurrence of BE after successful CEIM. Prior studies in general lacked statistical power to assess predictors of recurrence. Vaccaro and colleagues demonstrated that patients with recurrent IM had longer BE segments at baseline than those who remained free of IM (median, $4 \mathrm{~cm}$ vs. $2 \mathrm{~cm}$; $\mathrm{p}=0.03$ ), similar to our finding. ${ }^{22}$ Orman et al. published data on recurrence of CEIM in patients with either baseline dysplastic BE or IMC treated with RFA and found no statistical difference in a variety of clinical, endoscopic, and treatment variables as predictors of recurrence. ${ }^{10}$ Unfortunately, this study was limited by a small number of overall recurrences and multivariate analysis could not be performed. The above data likely suffer from type II error due to relatively small sample sizes and the few patients who suffered recurrence. In contrast, given the large cohort in the present study, we were able to perform both bivariate and multivariate analysis to determine a number of predictors of recurrence which can be used to guide clinical practice.

Our study has limitations that must be considered when interpreting our data. Our study was strictly observational and we could not mandate care paradigms. It is possible that physicians did not follow the recommended biopsy protocol. Additionally, TMN staging for cancers was not provided in the registry. Given the size and nature of our study, there was no central lab for re-interpretation of pathological specimens, thus interobserver variation almost certainly introduces misclassification into our study. ${ }^{23,24}$ However, nationwide, few clinicians have access to highly specialized labs for biopsies, and our results are likely a better reflection of real-world outcomes than tertiary center reports. While the majority of 
the patients (66\%) in our cohort were enrolled prospectively, the retrospective portion of this study also makes misclassification error possible. However, assessment of our primary outcome in subjects with solely prospective data collection shows no meaningful difference between recurrence rates (19.8\% with only prospective data vs. $20.4 \%$ for all subjects). Furthermore, random esophageal biopsies performed after CEIM is attained may underestimate the prevalence of residual or recurrent IM, especially sub-squamous IM.

Additionally, the $20 \%$ IM recurrence rate may be a low-end estimation given our protocol did not require that routine biopsies be taken at the gastro-esophageal junction. We also excluded the $2 \%$ of patients that had empiric RFA re-treatment without histologic confirmation since we are unable to confirm whether these patients had recurrent metaplasia. Finally, some subjects identified as having IM recurrence may have instead had incomplete initial eradication of IM and would thus actually be failures of initial therapy, rather than true recurrences.

Our study has several strengths. We report the largest cohort of patients treated with RFA for $\mathrm{BE}$ and the largest reported study assessing durability and predictors of CEIM. This study used the U.S. RFA Patient Registry, which includes 320 physician participants from 148 institutions, both community-based and academic-affiliated. The diversity of practice settings and variety of procedural volumes improves the external validity of our results. Additionally, study definitions were $a$ priori, and data were collected in a standardized fashion.

Our findings have implications on future endoscopic surveillance intervals following ablation. Given that the mean time to IM recurrence in patients with NDBE or IND at baseline was 2.1 and 1.9 years, respectively, and that none of these patients progressed to cancer, patients with NDBE or IND at pre-treatment do not appear to require initial surveillance endoscopies at intervals $<2$ years. On the other hand, more frequent surveillance may be warranted in patients who are older, and had longer pre-treatment segments. Additionally, non-Caucasians were found to have increased risk of IM recurrence. Although the reasons for these associations remain unclear, it is possible that elderly people have more prolonged exposure to carcinogens and are more likely to accumulate somatic mutations, while a longer pre-treatment segment may be a marker for more severe acid exposure and injury. Perhaps non-Caucasians are at increased risk because of a genetic predisposition to recurrence or from socio-economic factors that may affect follow-up appointments, treatment, or medication adherence.

In conclusion, after successful treatment of BE with RFA, the majority of patients demonstrate durability of squamous epithelium. However, regardless of the definition of disease eradication used, approximately $1 / 5^{\text {th }}$ of patients followed for a mean of 2.4 years had recurrent BE. When BE did recur, most recurrences were of short length and either nondysplastic or indefinite for dysplasia. The likelihood for recurrence after successful treatment with RFA was increased in those who are older, not Caucasian, and had longer BE segments. Additionally, more severe baseline histology predicts recurrence in Kaplan-Meier estimates, although this finding is equivocal when controlled for other patient characteristics. These data suggest that continued surveillance following treatment is necessary to detect recurrent BE. Additionally, we suggest attenuation of surveillance 
intervals based on these data. Finally, we have identified specific factors that predict recurrence of IM, which can be used to better stratify risk and personalize follow-up surveillance.

\section{Supplementary Material}

Refer to Web version on PubMed Central for supplementary material.

\section{Acknowledgments}

This research was funded by T32 DK07634 from the National Institutes of Health and GI Solutions, a subsidiary of Covidien.

Relevant Financial Disclosures: Dr. Shaheen receives research funding from Covidien Medical, CSA Medical, NeoGenomics, Takeda Pharmaceuticals and Oncoscope. He is a consultant for Oncoscope.

\section{References}

1. Ronkainen J, Aro P, Storskrubb T, et al. Prevalence of Barrett's esophagus in the general population: an endoscopic study. Gastroenterology. 2005; 129:1825-31. [PubMed: 16344051]

2. Rastogi A, Puli S, El-Serag HB, et al. Incidence of esophageal adenocarcinoma in patients with Barrett's esophagus and high-grade dysplasia: a meta-analysis. Gastrointest Endosc. 2008; 67:3948. [PubMed: 18045592]

3. Zagari RM, Fuccio L, Wallander MA, et al. Gastro-oesophageal reflux symptoms, oesophagitis and Barrett's oesophagus in the general population: the Loiano-Monghidoro study. Gut. 2008; 57:13549. [PubMed: 18424568]

4. Pohl H, Welch HG. The role of overdiagnosis and reclassification in the marked increase of esophageal adenocarcinoma incidence. J Natl Cancer Inst. 2005; 97:142-6. [PubMed: 15657344]

5. Lepage C, Rachet B, Jooste V, et al. Continuing rapid increase in esophageal adenocarcinoma in England and Wales. Am J Gastroenterol. 2008; 103:2694-9. [PubMed: 18853967]

6. Bulsiewicz WJ, Shaheen NJ. The role of radiofrequency ablation in the management of Barrett's esophagus. Gastrointest Endosc Clin N Am. 2011; 21:95-109. [PubMed: 21112500]

7. Lyday WD, Corbett FS, Kuperman DA, et al. Radiofrequency ablation of Barrett's esophagus: outcomes of 429 patients from a multicenter community practice registry. Endoscopy. 2010; 42:272-8. [PubMed: 20146164]

8. Sharma VK, Jae Kim H, Das A, et al. Circumferential and focal ablation of Barrett's esophagus containing dysplasia. Am J Gastroenterol. 2009; 104:310-7. [PubMed: 19174783]

9. Shaheen NJ, Sharma P, Overholt BF, et al. Radiofrequency ablation in Barrett's esophagus with dysplasia. N Engl J Med. 2009; 360:2277-88. [PubMed: 19474425]

10. Orman ES, Kim HP, Bulsiewicz WJ, et al. Intestinal metaplasia recurs infrequently in patients successfully treated for Barrett's esophagus with radiofrequency ablation. Am J Gastroenterol. 2013; 108:187-95. quiz 196. [PubMed: 23247578]

11. Pouw RE, Gondrie JJ, Sondermeijer CM, et al. Eradication of Barrett esophagus with early neoplasia by radiofrequency ablation, with or without endoscopic resection. J Gastrointest Surg. 2008; 12:1627-36. discussion 1636-7. [PubMed: 18704598]

12. Fleischer DE, Overholt BF, Sharma VK, et al. Endoscopic radiofrequency ablation for Barrett's esophagus: 5-year outcomes from a prospective multicenter trial. Endoscopy. 2010; 42:781-9. [PubMed: 20857372]

13. Herrero LA, van Vilsteren FG, Pouw RE, et al. Endoscopic radiofrequency ablation combined with endoscopic resection for early neoplasia in Barrett's esophagus longer than $10 \mathrm{~cm}$. Gastrointest Endosc. 2011; 73:682-90. [PubMed: 21292262] 
14. Pouw RE, Wirths K, Eisendrath P, et al. Efficacy of radiofrequency ablation combined with endoscopic resection for barrett's esophagus with early neoplasia. Clin Gastroenterol Hepatol. 2010; 8:23-9. [PubMed: 19602454]

15. Shaheen NJ, Overholt BF, Sampliner RE, et al. Durability of radiofrequency ablation in Barrett's esophagus with dysplasia. Gastroenterology. 2011; 141:460-8. [PubMed: 21679712]

16. Wang KK, Sampliner RE. Gastroenterology PPCotACo. Updated guidelines 2008 for the diagnosis, surveillance and therapy of Barrett's esophagus. Am J Gastroenterol. 2008; 103:78897. [PubMed: 18341497]

17. Voltaggio L, Montgomery EA, Lam-Himlin D. A clinical and histopathologic focus on Barrett esophagus and Barrett-related dysplasia. Arch Pathol Lab Med. 2011; 135:1249-60. [PubMed: 21970480]

18. Shaheen NJ, Kim HP, Bulsiewicz WJ, et al. Prior fundoplication does not improve safety or efficacy outcomes of radiofrequency ablation: results from the U.S. RFA Registry. J Gastrointest Surg. 2013; 17:21-8. discussion p.28-9. [PubMed: 22965650]

19. Orman ES, Li N, Shaheen NJ. Efficacy and Durability of Radiofrequency Ablation for Barrett's Esophagus: Systematic Review and Meta-analysis. Clin Gastroenterol Hepatol. 2013

20. Phoa KN, Pouw RE, van Vilsteren FG, et al. Remission of Barrett's esophagus with early neoplasia 5 years after radiofrequency ablation with endoscopic resection: a Netherlands cohort study. Gastroenterology. 2013; 145:96-104. [PubMed: 23542068]

21. Haidry RJ, Dunn JM, Butt MA, et al. Radiofrequency ablation and endoscopic mucosal resection for dysplastic barrett's esophagus and early esophageal adenocarcinoma: outcomes of the UK National Halo RFA Registry. Gastroenterology. 2013; 145:87-95. [PubMed: 23542069]

22. Vaccaro BJ, Gonzalez S, Poneros JM, et al. Detection of intestinal metaplasia after successful eradication of Barrett's Esophagus with radiofrequency ablation. Dig Dis Sci. 2011; 56:19962000. [PubMed: 21468652]

23. Reid BJ, Haggitt RC, Rubin CE, et al. Observer variation in the diagnosis of dysplasia in Barrett's esophagus. Hum Pathol. 1988; 19:166-78. [PubMed: 3343032]

24. Montgomery E, Bronner MP, Goldblum JR, et al. Reproducibility of the diagnosis of dysplasia in Barrett esophagus: a reaffirmation. Hum Pathol. 2001; 32:368-78. [PubMed: 11331953] 


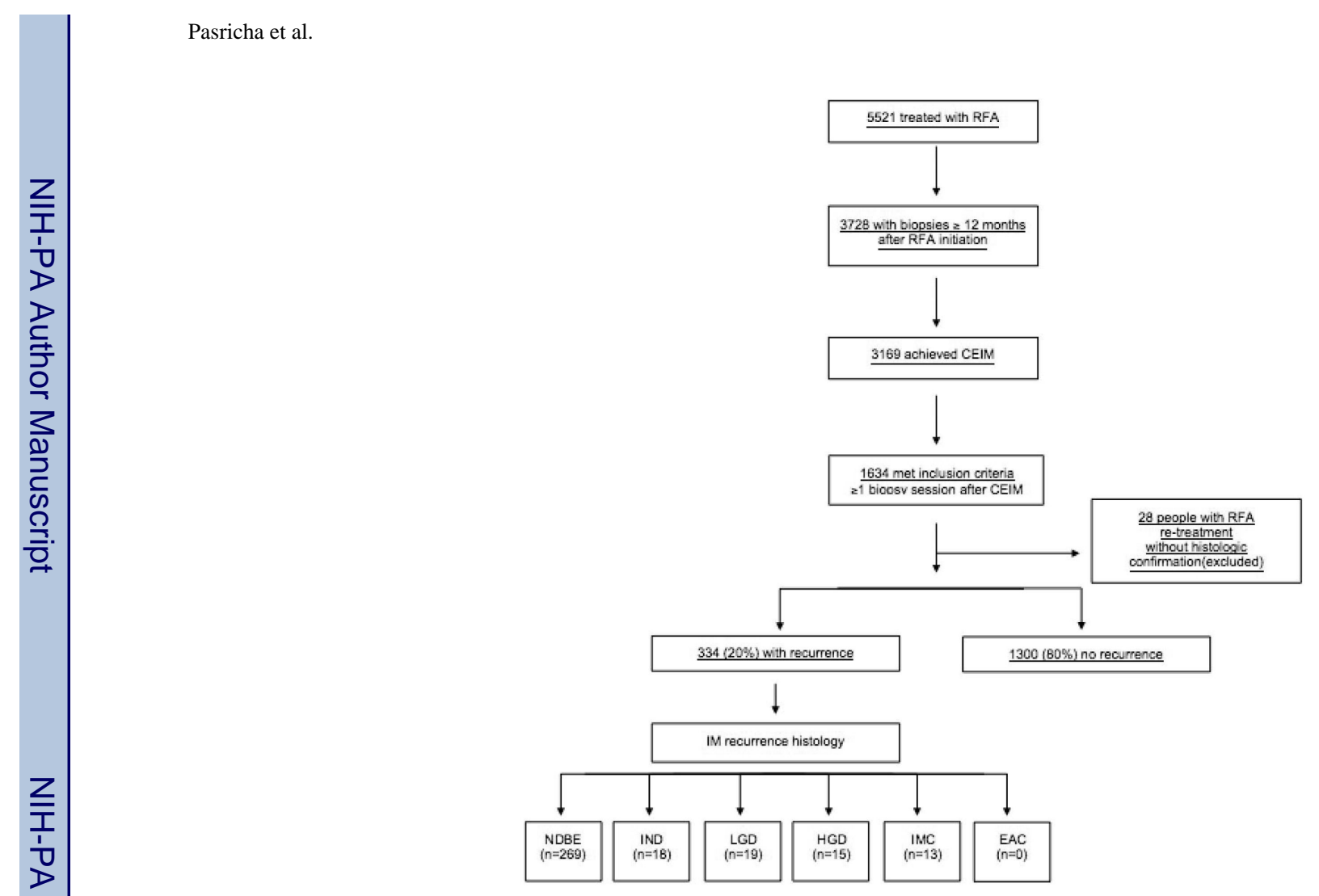

Figure 1.

Flow diagram of patient enrollment 


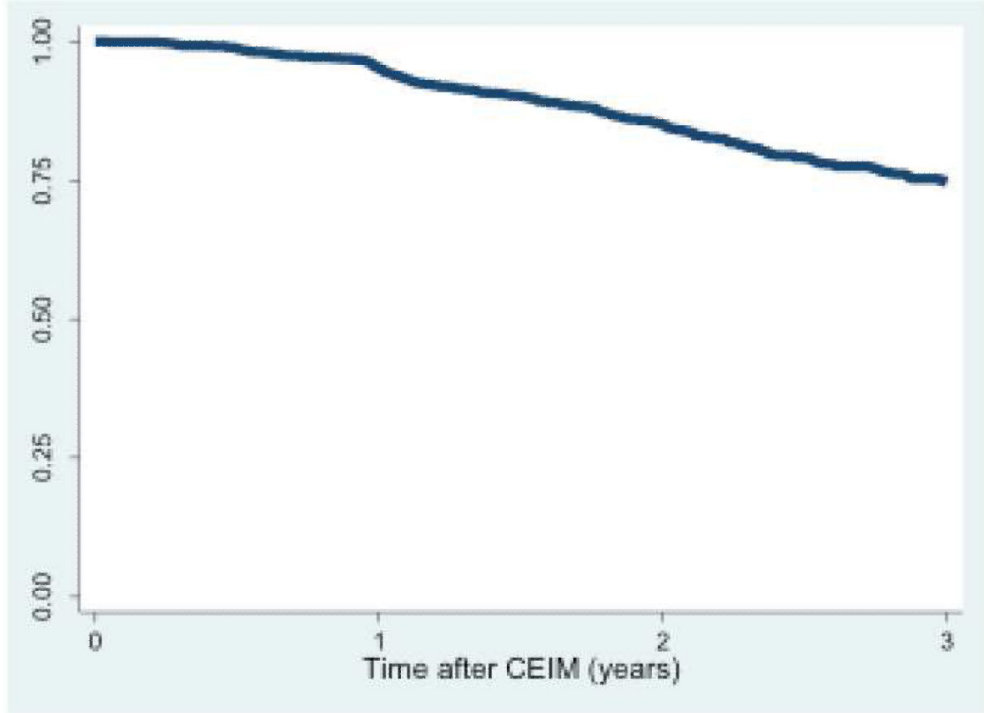

Figure 2a. Recurrence free survival in all study subjects $(n=1634)$

Kaplan-Meier plot of IM recurrence among patients who achieved CEIM after RFA, overall. 


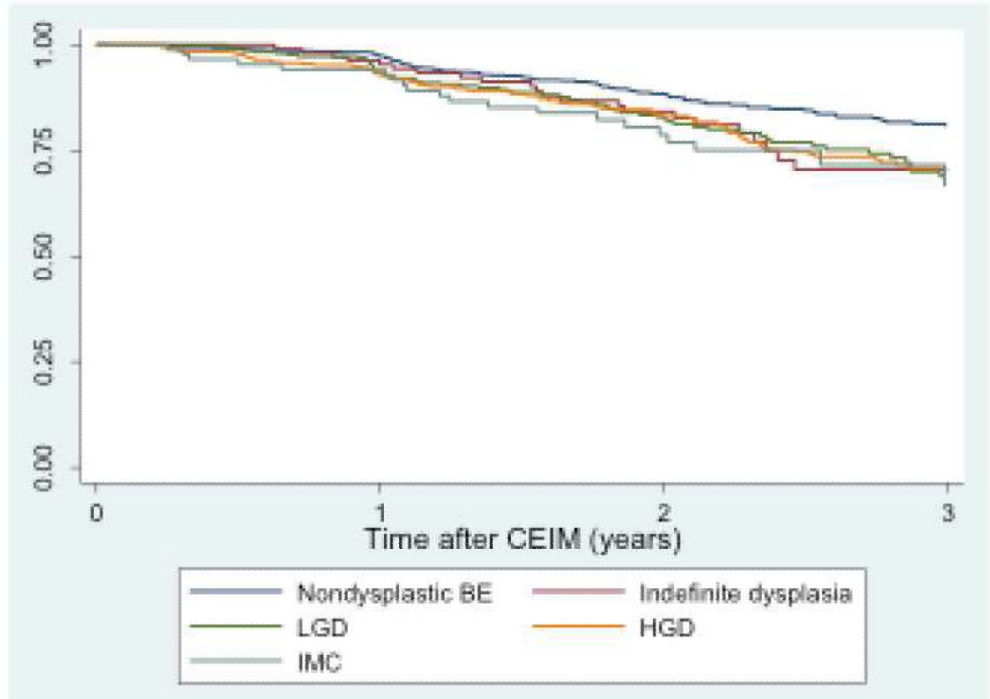

Figure 2b. Recurrence free survival study subjects grouped by pre-treatment histology ( $\mathrm{n}=\mathbf{1 6 1 3}$ ) Kaplan-Meier plot of IM recurrence among patients who achieved CEIM after RFA, with pre-treatment histology nondysplasic BE, low-grade dysplasia (LGD), and high-grade dysplasia (HGD). $\mathrm{P}=0.001$ between dysplastic and non-dysplastic BE. 


\section{Table 1}

Baseline characteristics of patients under endoscopic surveillance following CEIM

\begin{tabular}{|c|c|}
\hline & $n=1634$ \\
\hline Age, mean years \pm SD & $61.7 \pm 10.9$ \\
\hline Male, $\mathrm{n}(\%)$ & $1204(74)$ \\
\hline \multicolumn{2}{|l|}{ Race, $\mathrm{n}(\%)$} \\
\hline Caucasian & $1517(93)$ \\
\hline Black & $27(2)$ \\
\hline Hispanic & $32(2)$ \\
\hline Asian & $11(1)$ \\
\hline Other/not identified & $47(3)$ \\
\hline \multicolumn{2}{|l|}{ Pre-Treatment histology, n (\%) } \\
\hline Nondysplastic BE & $668(41)$ \\
\hline Indefinite Dysplasia & $114(7)$ \\
\hline Low-grade dysplasia & $323(20)$ \\
\hline High-grade dysplasia & $416(25)$ \\
\hline Carcinoma & $113(7)$ \\
\hline Time of Observation in years, mean $\pm \mathrm{SD}$ & $2.4 \pm 1.3$ \\
\hline Length of BE segment, mean $\mathrm{cm} \pm \mathrm{SD}$ & $4.0 \pm 3.1$ \\
\hline Fundoplication before treatment, $\mathrm{n}(\%)$ & $95(6)$ \\
\hline EMR before RFA, n (\%) & $212(13)$ \\
\hline RFA treatment sessions, mean \pm SD & $2.9 \pm 1.7$ \\
\hline Circumferential RFA session, mean \pm SD & $0.7 \pm 0.9$ \\
\hline Focal RFA sessions, mean \pm SD & $2.2 \pm 1.5$ \\
\hline Biopsy sessions performed after CEIM, mean \pm SD & $4.3 \pm 1.8$ \\
\hline Treated at academic medical center, $\mathrm{n}(\%)$ & $536(33)$ \\
\hline
\end{tabular}


Table 2

IM recurrence rate stratified by pre-treatment histology

\begin{tabular}{|l|c|c|c|c|c|c|c|c|}
\hline & \multicolumn{7}{|c|}{ Pre-treatment Histology } \\
\hline & $\begin{array}{c}\text { All } \\
\text { patients } \\
(\mathbf{N = 1 6 3 4})\end{array}$ & $\begin{array}{c}\text { NDBE } \\
(\mathbf{N = 6 6 8})\end{array}$ & $\begin{array}{c}\text { IND } \\
(\mathbf{N = 1 1 4})\end{array}$ & $\begin{array}{c}\text { LGD } \\
(\mathbf{N}=\mathbf{3 2 3})\end{array}$ & $\begin{array}{c}\text { HGD } \\
(\mathbf{N = 4 1 6})\end{array}$ & $\begin{array}{c}\text { IMC } \\
(\mathbf{N = 9 2})\end{array}$ & $\begin{array}{c}\text { EAC } \\
(\mathbf{N = 2 1})\end{array}$ & $\begin{array}{c}\text { p- } \\
\text { value }\end{array}$ \\
\hline $\begin{array}{l}\text { \# Recurrences, } \\
\text { n (\%) }\end{array}$ & $334(20)$ & $119(18)$ & $25(22)$ & $70(22)$ & $93(22)$ & $21(23)$ & $6(29)$ & \\
\hline $\begin{array}{l}\text { Time to } \\
\text { recurrence, } \\
\text { years; mean } \pm \\
\text { SD }\end{array}$ & $1.9 \pm 1.1$ & $2.1 \pm 1.2$ & $1.9 \pm 1.0$ & $1.8 \pm 1.0$ & $1.7 \pm 1.1$ & $1.5 \pm 0.9$ & $1.4 \pm 0.8$ & 0.11 \\
\hline $\begin{array}{l}\text { Recurrences/ } \\
\text { year }\end{array}$ & $9 \%$ & $7 \%$ & $10 \%$ & $11 \%$ & $10 \%$ & $12 \%$ & $19 \%$ & 0.0003 \\
\hline
\end{tabular}


Table 3

Histology of patients with IM recurrence stratified by pre-treatment histology

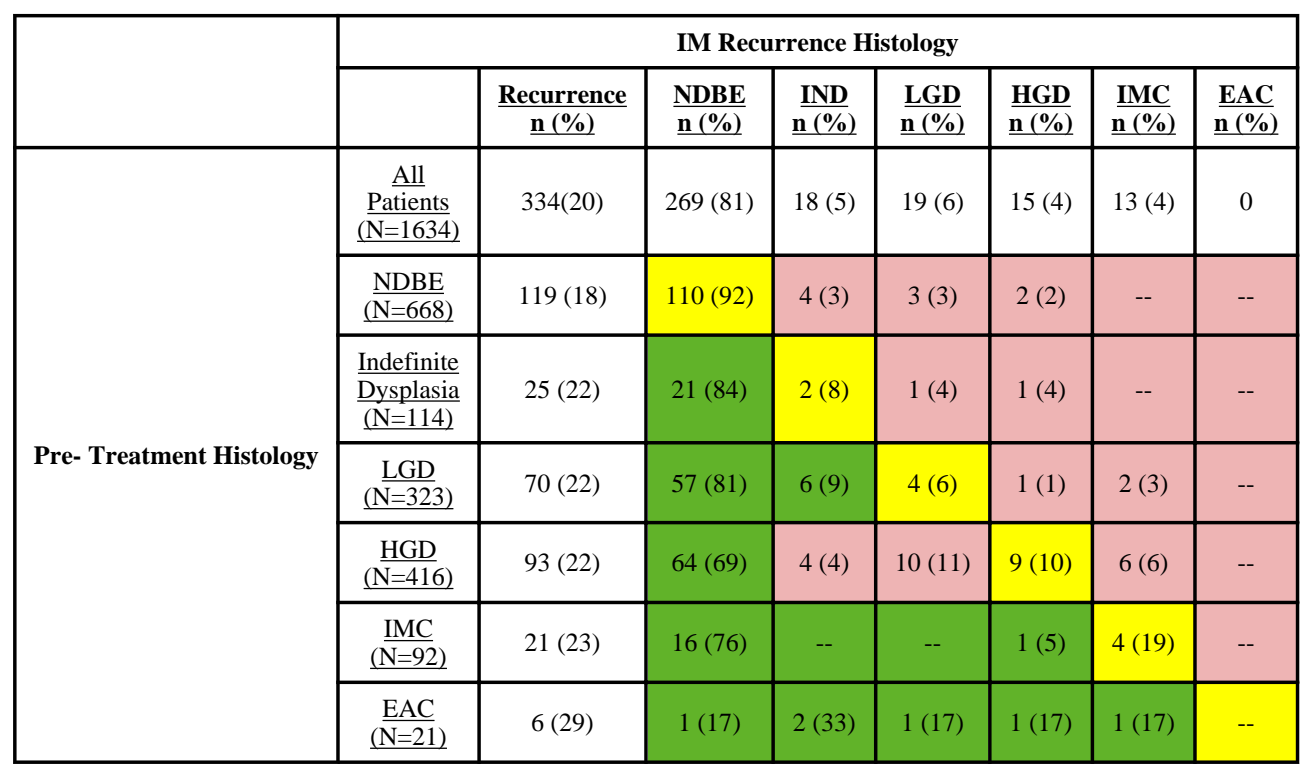

Color coding: Green, less severe histology at time of IM recurrence; Yellow, same pre and post histology at time of IM recurrence; Red, more severe histology at time of IM recurrence. 
Table 4

Multivariate Analysis of Predictors of Recurrence

\begin{tabular}{|l|c|c|}
\hline Odds Ratio $(95 \%$ CI $)$ & Base Model & Reduced Model \\
\hline Age, per year & $1.02(1.01-\mathbf{1 . 0 3})$ & $1.02(1.01-\mathbf{1 . 0 3})$ \\
Not Caucasian & $\mathbf{2 . 0 9}(\mathbf{1 . 2 4}-\mathbf{3 . 5 1})$ & $\mathbf{2 . 0 0}(\mathbf{1 . 2 0}-\mathbf{3 . 3 4})$ \\
Male gender & $\mathbf{1 . 1 6}(\mathbf{0 . 8 6}-\mathbf{1 . 5 7})$ & -- \\
Length of BE segment, per cm & $\mathbf{1 . 0 9}(\mathbf{1 . 0 4}-\mathbf{1 . 1 4})$ & $\mathbf{1 . 1 0}(\mathbf{1 . 0 6}-\mathbf{1 . 1 5})$ \\
Pre-treatment fundoplication & $\mathbf{1 . 4 2}(\mathbf{0 . 8 7}-\mathbf{2 . 3 2})$ & -- \\
Pretreatment dysplastic BE & $\mathbf{1 . 0 8}(\mathbf{0 . 8 1}-\mathbf{1 . 4 4})$ & -- \\
Treated with EMR before RFA & $\mathbf{0 . 9 5}(\mathbf{0 . 6 4}-\mathbf{1 . 4 1})$ & -- \\
Total RFA sessions, per treatment & $\mathbf{1 . 0 4}(\mathbf{0 . 9 6}-\mathbf{1 . 1 2})$ & -- \\
Treatment at community practice & $\mathbf{1 . 0 4}(\mathbf{0 . 7 8}-\mathbf{1 . 3 8})$ & -- \\
\hline
\end{tabular}

Reduced Model includes age, race, and length of BE segment. 